adversely affect home-based male partner HIV testing during pregnancy among 260 men, as HIV test uptake was $96 \%$ before (of 180), 95\% after (of 80) syphilis test introduction, and remained 2-times greater than clinic-based HIV testing alone within the RCT (39\%). Finally, men intended to seek clinic treatment if they received a positive test result during pregnancy and postpartum (94\% and 95\%, respectively).

Conclusion Men were likely to accept both syphilis and HIV tests when offered at home without adversely affecting HIV testing approaches. POC diagnostics can work well outside facilities and increase testing of male partners who rarely accompany women to antenatal clinics.

\section{P3.107 RATES OF PRIMARY AND SECONDARY SYPHILIS BY STATE AND RACE/ETHNICITY AMONG MEN WHO HAVE SEX WITH MEN: UNITED STATES, 2014}

${ }^{1}$ Jeremy A Grey, ${ }^{2}$ Hillard Weinstock, ${ }^{2}$ Sarah Kidd, ${ }^{1}$ Eli S Rosenberg, ${ }^{2}$ Thomas L Gift, ${ }^{2}$ Kyle T Bernstein. ${ }^{1}$ Emory University, Atlanta, USA; ${ }^{2}$ Centres for Disease Control and Prevention, Atlanta, USA

\subsection{6/sextrans-2017-053264.342}

Introduction Men who have sex with men (MSM) accounted for $61.1 \%$ of reported primary and secondary ( $\mathrm{P}$ and $\mathrm{S}$ ) syphilis cases in the United States in 2014. Of P and S syphilis cases among MSM with known race/ethnicity, 94.7\% were either White (40.3\%), Black (32.2\%), or Hispanic $(22.2 \%)$. To examine rates of $\mathrm{P}$ and $\mathrm{S}$ syphilis among racial/ethnic categories of MSM, national and state-level estimates of the number of MSM of each race/ethnicity are needed.

Methods We calculated race/ethnicity-specific rates of $\mathrm{P}$ and $\mathrm{S}$ syphilis among adult MSM (age $\geq 18$ ) in 49 states that reported sex of partners and race/ethnicity for syphilis cases in 2014. Case counts of $P$ and $S$ syphilis were from national case report data, which are submitted from states to CDC. For rate denominators, we amended our previously published method to produce stratified estimates and 95\% confidence intervals (CI) for seven racial/ethnic groups: Hispanic MSM of any race and non-Hispanic MSM who were White; Black; American Indian; Asian; Pacific Islander; or multiple races.

Results The rate of reported P and S syphilis among MSM in the US was 255.4 (95\% CI: 229.1-284.7) per 100000 in 2014. The rates of $\mathrm{P}$ and $\mathrm{S}$ syphilis per 100000 among the three racial/ethnic groups most represented among MSM cases were 170.0 (151.1-191.0) for White MSM, 286.4 (250.1329.5) for Hispanic MSM, and 604.3 (525.0-700.8) for Black MSM, the highest of all racial/ethnic groups. Asian MSM had the lowest rate at $106.9(91.4-125.4)$ per 100000 . Comparing the 3 most-represented racial/ethnic groups, the rate was highest among Black MSM in 37 states, Hispanic MSM in 7 states, and White MSM in 5 states. Of states with $\geq 100$ cases among Black MSM, South Carolina had the highest rate at 1,398.1 (941.6-2,119.0) per 100000.

Conclusion These are the first race/ethnicity-specific estimates of $\mathrm{P}$ and $\mathrm{S}$ syphilis rates among MSM for states with reported sexual behaviour of cases. Although more cases of $\mathrm{P}$ and $\mathrm{S}$ syphilis were reported among White MSM in 2014, the rate among Black MSM was higher than White or Hispanic MSM in most states and was over 3.5 times that of White MSM in the US.

\section{P3.108 ANTIRETROVIRAL THERAPY USE DURING PREGNANCY AND ADVERSE BIRTH OUTCOMES AMONG HIV- INFECTED WOMEN IN LOW AND MIDDLE-INCOME COUNTRIES: A SYSTEMATIC REVIEW}

Jessica Londeree Saleska, Jesse Kwiek, Abigail Norris-Turner, Courtney Maierhofer. The Ohio State University, Columbus, USA

\subsection{6/sextrans-2017-053264.343}

Introduction Children born to HIV-infected women are at increased risk for adverse birth outcomes including preterm birth (PTB) and low birthweight (LBW). While antiretroviral therapy (ART) during pregnancy drastically reduces risk of vertical HIV transmission, LBW and PTB among HIV-exposed infants remains elevated. Exposure to certain ART regimens in utero may increase risk of adverse birth outcomes, in particular protease inhibitor (PI)-based regimens. Given the high burden of LBW and PTB in low- and middle-income countries, and efforts to increase ART use by HIV-infected pregnant women, it is critical to understand the precise effects of ART on adverse birth outcomes.

Methods We conducted a systematic review of the effects of different ART regimens used during pregnancy on LBW or PTB in low and middle income-countries. We searched electronic databases Medline, COCHRANE, Web of Science and SCOPUS, and CPCI-S for relevant papers published on or before 10 April 2016.

Results Our final review included 19 studies and assessed many ART regimens. Results were often heterogeneous. We observed no clear pattern for the effect of PI-based highly active antiretroviral therapy (HAART) on PTB compared to no therapy, or compared to non-PI-based HAART. We similarly saw no clear trends for the effect of non PI-based HAART on LBW compared to no therapy. In contrast, PIbased HAART was generally protective against LBW when compared to non-PI-based HAART and no therapy, and non PI-based HAART was generally associated with an increased risk of LBW when compared to monotherapy. Results were similar in unadjusted studies and those that controlled for maternal disease severity and other confounders.

Conclusion There is a wide array of ART regimens used by HIV-positive pregnant women in low- and middle-income countries, as well as the heterogeneity of results related to the adverse birth outcomes of PTB and LBW. Nonetheless, we found that PI-based HAART was generally protective against LBW when compared to non-PI-based HAART.

\section{P3.109 POINT-OF-CARE TESTING FOR SEXUALLY TRANSMITTED INFECTIONS IN HIV PREVENTION TRIALS}

1J Naidoo, ${ }^{1} \mathrm{~N}$ Samsunder, ${ }^{1} \mathrm{~K}$ Govender, ${ }^{1} \mathrm{R}$ Noble, ${ }^{1} \mathrm{~N}$ Ngubane, ${ }^{1} \mathrm{~N}$ Naicker, ${ }^{1} \mathrm{~A}$ Mindel, ${ }^{2} \mathrm{~A}$ Rompalo, ${ }^{1} \mathrm{~K}$ Mngadi, ${ }^{1} \mathrm{~L}$ Mansoor, ${ }^{1} \mathrm{~N}$ Garrett. ${ }^{1}$ Centre for the AIDS Programme of Research in South Africa (CAPRISA), Durban, South Africa; ${ }^{2}$ Johns Hopkins University, Baltimore, USA

\subsection{6/sextrans-2017-053264.344}

Introduction Accurate HIV risk assessment is essential when screening volunteers for HIV prevention studies. STI testing plays a key role, but has traditionally been conducted in central laboratories resulting in reporting delays, which can impact on screening and enrolment decisions, and on participant care during follow-up. Here, we outline the 